\title{
Joint Model for Fine Synchronization and Adaptive LMMSE Channel Estimation in Uplink OFDMA
}

\author{
Kamran Khan*†, Andreas Ibing ${ }^{\dagger \dagger}$, Dirk Dahlhaus* \\ ${ }^{*}$ Communications Laboratory, University of Kassel, Wilhelmshöher Allee 73, 34121 Kassel, Germany \\ ${ }^{\dagger}$ Fraunhofer Institute for Telecommunications, Heinrich-Hertz-Institut, Einsteinufer 37, 10587 Berlin, Germany \\ ${ }^{\ddagger}$ Heinrich-Hertz-Chair for Mobile Communications, Technical University of Berlin, Einsteinufer 25, 10587 Berlin, Germany
}

\begin{abstract}
In this paper parametric tracking of channel and synchronization errors for an adaptive multi-dimensional channel estimator for uplink Orthogonal Frequency Division Multiplex Access (OFDMA) communication systems is proposed. The estimator consists of a two-dimensional LMMSE filter. In real world scenarios, channel estimation should work not only in a wide range of terminal velocities and delay spread but it is also confronted with time and frequency synchronization errors affecting the channel estimation and thus limits the performance of both the coherent receiver and overall OFDMA system. We jointly track channel statistics and synchronization errors so that LMMSE filter coefficients closely reflect the current statistics of the channel. Through simulations it is verified that the proposed channel estimator outperforms the robust LMMSE estimator in the presence of synchronization errors and varying channel conditions.

Index Terms-Adaptive Wiener filtering, channel tracking, synchronization
\end{abstract}

\section{INTRODUCTION}

OFDMA is used widely in current communication systems due to bandwidth efficiency, multipath immunity and achievable high data rates [1]. In wireless communications, the mobile radio channel between transmitter and receiver causes time and frequency selective fading. For receivers using coherent demodulation, channel estimation is required. Higher performance channel estimation depends upon the estimation of both these fading processes. Practical communication systems are also confronted by time and frequency synchronization errors. Time synchronization error also referred as beginning of frame offset (BOF) is caused by mismatch of frame sampling instant at the transmitter and receiver due to propagation delays [2] while frequency synchronization error also referred as carrier frequency offset (CFO) is caused by difference between transmitter and receiver oscillator frequencies as well as Doppler effects [3].

The necessary estimation of 2D (time and frequency) stochastic channel can be performed by inserting pilot symbols in the data stream in a pattern which is known at the receiver. At the receiver the sampled time variant transfer function (TVTF) of the channel is obtained using least squares filtering at the pilot positions. If the sampling criterion is fulfilled, the TVTF of the channel can be estimated

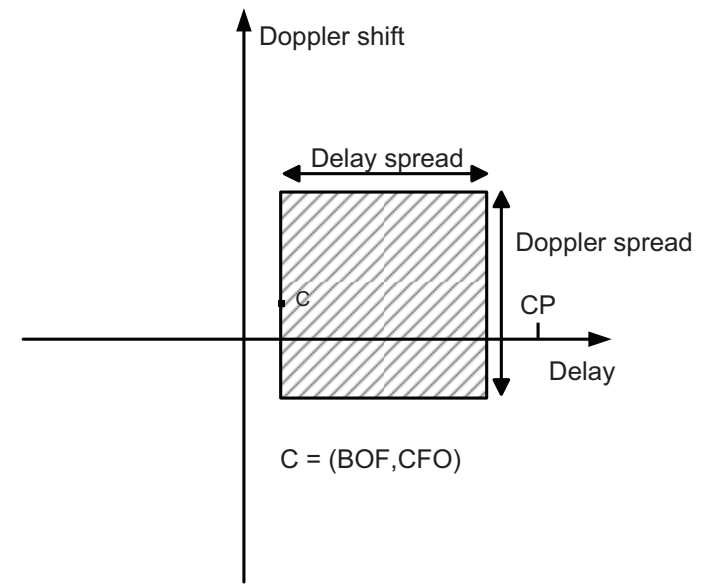

Fig. 1. 4- Parametric model of 2D power spectral density (scattering function) assuming uniform distribution.

using 2D linear minimum mean square error (LMMSE) filter also known as Wiener interpolation filter [4]. LMMSE filter is the optimal estimator in mean square error (MSE) sense when channel autocorrelation and noise variance are known [5]. Depending upon the complexity and performance required, a static or adaptive version of LMMSE filter is employed at the receiver. A static LMMSE filter is based on robust filter design using worst case assumptions of maximum delay spread and Doppler spread so it assumes less autocorrelation between the pilots than there is typically [6], while an adaptive LMMSE filter tracks the channel statistics to exploit as much correlation between the pilots as possible [7].

LMMSE filters designed using only wireless channel statistics without taking the synchronization issues under consideration perform only sub-optimally [8]. So in this paper we extend the 2-parameter tracking model as in [7] to a 4-parameter tracking model and propose an adaptive 2D LMMSE estimator which performs optimally even in the presence of synchronization offsets. We track a parametric model as illustrated in Fig. 1 and then use it to adapt LMMSE filter coefficients to current statistics of the channel. 
The width of the window in the Fig. 1 indicates delay spread, the height depicts Doppler spread and the location of the point $C$ of the window show BOF and CFO.

The organization of the paper is as follows: In section II system model and robust (static) LMMSE channel estimation are discussed. Then the parametric tracking for adaptive LMMSE channel estimation is discussed in section III. Section IV and V concludes the paper by simulation results and some discussion.

\section{Review of Robust Static Filtering}

We use OFDMA transmitter with length $N$ IFFT such that $N-N_{\text {subcar }}$ subcarriers are guard band and $N_{\text {subcar }}$ are data carrying subcarriers. Transmitter is assumed to be using subband carrier allocation scheme. If each user is represented by $m$ and total number of users are $M(m=1 \ldots M)$ where $M \ll N_{\text {subcar }}$ is assumed, only $s=N_{\text {subcar }} / M$ subcarriers can be assigned to a user for transmission when all users are active. Let $x_{m}=\left[\begin{array}{ll}x_{m, c p} & x_{m, \text { data }}\end{array}\right]^{T}$ denote the time domain representation of a OFDMA symbol for a user $m$ where $x_{m, \text { data }}=\left(x_{m, 1} x_{m, 2} \ldots x_{m, N}\right)$ are data symbols and $x_{m, c p}$ is the cyclic prefix which consist of last $L$ symbols of $x_{m \text {,data }}$ to avoid inter-symbol interference. We use wide sense stationary uncorrelated scattering Rayleighfading channel model. The received signal at the base station is the superposition of signals from all active users. Let the received signal after cyclic prefix removal, FFT and then guard band removal be denoted by $Y$.

Now, Representing $n$ as subcarriers and $k$ as OFDMA symbol index, the discrete baseband model of the received signal in the frequency domain in matrix form for a user $m$ is given by:

$$
Y_{m}[n, k]=X_{m}[n, k] H_{m}[n, k]+Z_{m}[n, k]
$$

where $Y_{m}[n, k]$ is the received signal, $X_{m}[n, k]$ is the transmitted signal, $H_{m}[n, k]$ is the TVTF of the channel, $Z_{m}[n, k]$ is the additive white gaussian noise and additional noise due to ICI caused by doppler spread and CFO. As a initial step to channel estimation, least squares filtering is applied at the pilot positions to obtain sampled TVTF corrupted with the noise:

$$
\tilde{H}_{m, p}[n, k]=\frac{Y_{m, p}[n, k]}{X_{m, p}[n, k]}=H_{m, p}[n, k]+\frac{Z_{m, p}[n, k]}{X_{m, p}[n, k]} .
$$

LMMSE filtering based on the initial least squares estimates is given by:

$$
\hat{H}_{m, p}[n, k]=\sum_{n_{0}, k_{0}} c^{*}\left[n_{0}, k_{0}\right] \tilde{H}_{m, p}\left[n-n_{0}, k-k_{0}\right]
$$

where $n_{0}$ and $k_{0}$ are the sampled locations within the filter range. $\boldsymbol{c}\left[n_{0}, k_{0}\right]$ are the LMMSE filter coefficients given by:

$$
\left.\boldsymbol{c}\left[n_{0}, k_{0}\right]\right|_{\text {opt }}=\left(\boldsymbol{R}[n, k]+\frac{\boldsymbol{I}}{S N R}\right)^{-1} \boldsymbol{r}[n, k]
$$

where $\boldsymbol{R}[n, k]$ is the autocorrelation between $H_{m}[n, k]$ at the pilots locations. $\boldsymbol{r}[n, k]$ is the crosscorrelation between

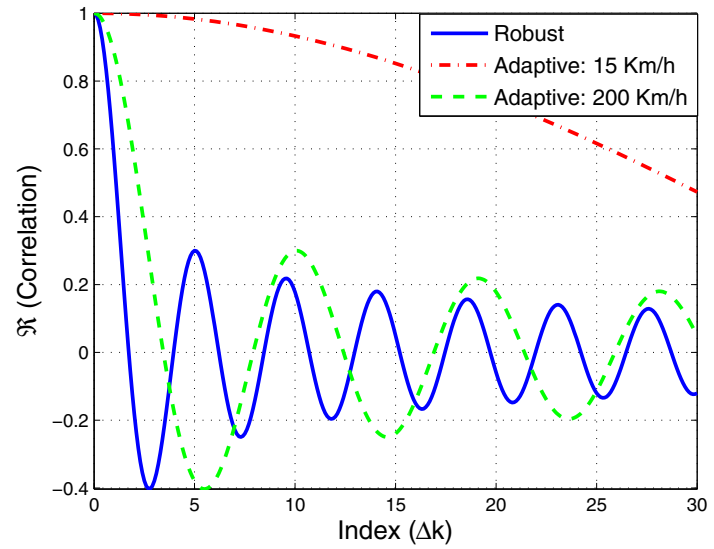

Fig. 2. Assumed time direction autocorrelation function for adaptive and robust filtering for operating frequency $2.1 \mathrm{GHz}$. LMMSE robust filter time direction autocorrelation function is designed for maximum velocity of 400 $\mathrm{km} / \mathrm{h}$.

$H_{m}[n, k]$ at the positions to be computed and the pilots. To obtain TVTF at the data locations, multidimensional up sampling interpolation is performed using noise suppressed (LMMSE filtered) channel estimates at the pilot positions.

The spaced-time, spaced-frequency (STSF) 2D correlation function of the channel is given by [9]:

$$
r_{H}(\Delta f, \Delta t)=E\left\{H_{m}(f, t) H_{m}^{*}(f+\Delta f, t+\Delta t)\right\}
$$

which is related to multidimensional power spectrum (scattering function) $S\left(\tau, f_{d}\right)$ of the channel (cf. Fig. 1 and Fig. 2) by:

$S\left(\tau, f_{d}\right)=\int_{\infty}^{\infty} \int_{\infty}^{\infty} r_{H}(\Delta f, \Delta t) e^{-j 2 \pi\left(f_{d} \Delta t-\tau \Delta f\right)} d \Delta t d \Delta f$

where $\tau$ represents delay and $f_{d}$ represents Doppler frequency. At the receiver, the actual shape of multidimensional power spectrum is generally not known. For the robust static filtering usually uniform multidimensional power spectrum is assumed [6].

STSF 2D correlation function can be separated into time and frequency parts:

$$
r_{H}(\Delta f, \Delta t)=\sigma_{H}^{2} r_{H}(\Delta f) r_{H}(\Delta t)
$$

where $\sigma_{H}^{2}$ denotes the average power which is assumed to be 1. For the uniform power spectrum case the correlation in time direction of the TVTF of the channel becomes [4]:

$$
r_{H, u n i f}(\Delta t)=\operatorname{sinc}\left(2 \pi f_{D} \Delta t\right)
$$

with $\operatorname{sinc}(x)=\sin (x) / x$, and $f_{D}$ being the Doppler spread. $\Delta t=\Delta k t_{s}(L+N)$ with $t_{s}$ being the sampling period and $t_{s}(L+N)$ one OFDM symbol duration. The correlation in frequency direction becomes [4]:

$$
r_{H, \text { unif }}(\Delta f)=\operatorname{sinc}\left(\pi \tau_{\max } \Delta f\right) e^{j 2 \pi \tau_{\text {shift } \Delta f}}
$$

with $\tau_{\max }$ as the delay spread, $\tau_{\text {shift }}=\frac{\tau_{\max }}{2}, \Delta f=$ $\Delta F \Delta n$ with $\Delta F$ being the subcarrier distance. Worst case 


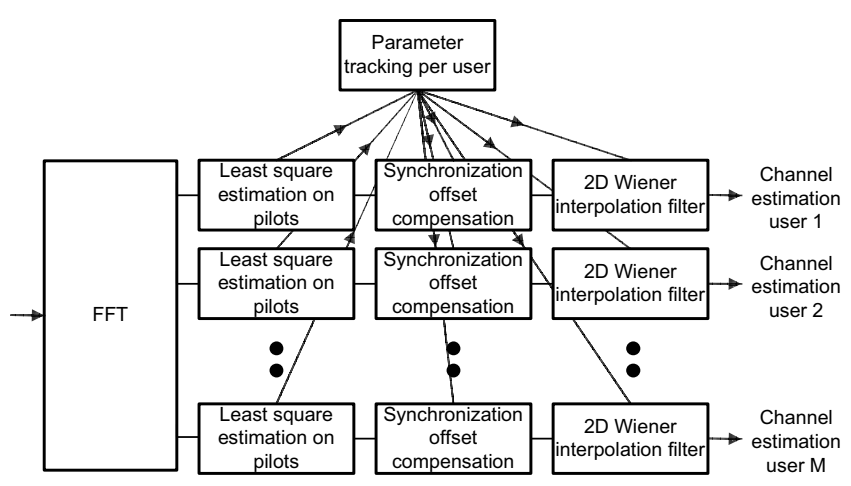

Fig. 3. Channel and synchronization offset parametric tracking and adaptive channel estimation.

channel characteristics assumptions $\left(\tau_{\max }=L t_{s}\right.$ and $f_{D}=$ maximum anticipated Doppler spread) leads to robust static LMMSE filtering [6].

\section{PARAmetric Multidimensional PSD TRACKING}

Robust filtering is less complex compared to adaptive filtering but its performance deteriorates as the model mismatch (difference between the correlation function that is assumed to generate filter coefficients to actual correlation function as in (5)) increases. To minimise model mismatch, channel parameters are tracked to exploit the available correlation between the pilots. Fig. 2 depicts the difference between available time direction correlation, present among the pilots for different user velocities for robust and adaptive filtering. Robust filtering always assumes minimum correlation among the pilots.

In the presence of synchronization offsets (CFO and $\mathrm{BOF})$, the problem becomes more interesting because synchronization errors shift the PSD (Power Spectral Density) as seen by the receiver introducing inter-carrier interference (ICI) and if $\mathrm{BOF}>L-\tau_{\max }$, bringing in also inter-symbol interference (ISI) which further worsens the robust LMMSE filter performance. In order to do optimal noise reduction filtering we jointly track the channel characteristics $\left(\tau_{\max }\right.$ and $\left.f_{D}\right)$ and synchronization errors for each user and then use them for filter adaptation and synchronization phase rotation compensation as shown in Fig. 3. Here it is important to note that unlike in downlink, in uplink each user's synchronization errors cannot be completely pre-compensated at the base station since it would require compensation before FFT operation which could miss align already aligned users. So only phase rotations due to remaining uncompensated synchronization errors are compensated at the base station while coarse synchronization adjustment is done through control channel at the user end by transmitting instructions (timing advance).

\section{A. BOF and Delay Spread Estimation}

BOF hinders in correctly extracting data symbols from the time domain OFDMA symbol and causes phase rotation of channel coefficients along the subcarriers in frequency domain if $\mathrm{BOF}<\left(L-\tau_{\max }\right)$, otherwise phase rotation is accompanied by ISI. It is assumed in the model that each user is already coarse synchronized so there would be only phase rotations:

$$
H_{m}[n, k]=H_{m}[n, k] \cdot \exp \left(\frac{j 2 \pi d_{m} n}{N}\right)
$$

where $d_{m}$ is integer BOF represented in number of samples. Using time-shift property of Fourier transform, (10) can be considered as shift in the power delay profile (PDP) of the channel. There are different techniques discussed in the literature for delay and delay spread estimation such as [10]- [13]. [14] has shown that synchronization errors bias the performance of delay spread estimators based on channel correlation. So we jointly estimate $d_{m}$ and $\tau_{\max }$ from the PDP without bias. PDP is calculated from the sampled TVTF of the channel:

$$
\begin{gathered}
h(\tau, t)=\mathcal{F} \mathcal{T}_{f \rightarrow \tau}\left\{H_{m, p}(f, t)\right\} \\
P_{h}(\tau) \approx \sum_{t}|h(\tau, t)|^{2}
\end{gathered}
$$

Where $P_{h}(\tau)$ represents the PDP of the channel for the user $m$. To avoid spectral leakage effects due to FFT, windowed version of $H_{m, p}(f, t)$ is used in (11). By comparing PDP against the threshold $(\delta), d_{m}$ and $\tau_{\max }$ are estimated by detecting first and last significant taps in $P_{h}(\tau)$ respectively. Threshold can be selected by:

$$
\delta=\left(\frac{K s}{L N}\right) \cdot \sum_{\tau=0}^{\left(\frac{L N}{s}\right)-1} P_{h}(\tau)
$$

where $K$ is a scaling factor.

\section{B. CFO and Doppler Spread Estimation}

Doppler spread is estimated using the time direction autocorrelation. To improve the estimation, time domain channel estimates are used for calculating the time direction autocorrelation as in [15]. It has been shown in [16] that for $3 \mathrm{D}$ scattering environments, Doppler spread follows the uniform distribution. The time direction autocorrelation of Rayleigh fading channel with uniform Doppler spectrum is a sinc function as in (8).

We search for the value of $\Delta t$ at which $r_{H, u n i f}(\Delta t)$ becomes $10 \%$ of its peak value which is equal to 1 . Estimate of $\Delta t$ is further refined through linear interpolation between the samples. $f_{D}$ is estimated using:

$$
f_{D} \approx \frac{2.852}{2 \pi \Delta t}
$$

Rather than using zero-crossing [17] or minimum extreme point of $r_{H, \text { unif }}(\Delta t)$ [7], $10 \%$ of the peak value of $r_{H, \text { unif }}(\Delta t)$ is selected as a reference point to calculate $f_{D}$ so that the Doppler spread estimation algorithm still remains functional (though with bad performance) even if the actual 


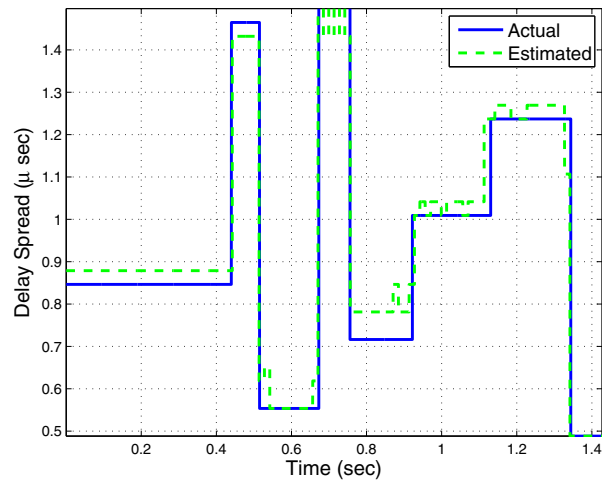

Fig. 4. Delay spread tracking.

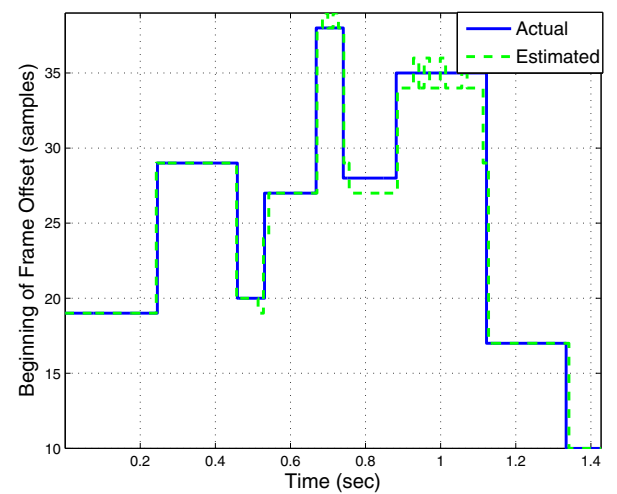

Fig. 5. Beginning of frame offset tracking.

shape of Doppler spectrum (whose autocorrelation might be a non-negative function for example in case of Gaussian distribution) differs to the assumed uniform Doppler spectrum.

CFO introduces common phase error (CPE) and ICI. CPE rotates each subcarrier by an equal amount while the effect of ICI is similar to noise [1]. CFO is estimated for each user by using maximum ratio combining similar formula:

$$
C P E=\frac{1}{2 \pi \Delta t} \cdot \angle
$$

where $\angle$ is given by:

$$
\angle=\arctan \left\{\frac{\sum_{n} \sum_{k} \Im\left(\hat{H}_{m, p}^{*}[n, k] \cdot \hat{H}_{m, p}[n, k+1]\right)}{\sum_{n} \sum_{k} \Re\left(\hat{H}_{m, p}^{*}[n, k] \cdot \hat{H}_{m, p}[n, k+1]\right)}\right\}
$$

\section{SNR Estimation}

SNR is adaptively estimated as in [7]. If $L>\tau_{\max }+d$ then most of the signal energy will gather in the interval $\tau_{\max }$ while rest of $\tau$ will contain only noise. So by adaptively estimating $\tau_{\max }$ and $d$ as in section III-A, SNR is estimated by dividing signal and noise power in the interval $\tau_{\max }$ to noise power in the rest of the duration $\tau$.

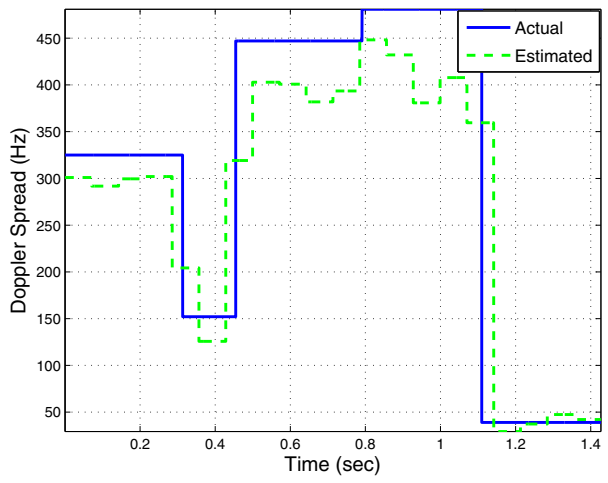

Fig. 6. Doppler spread tracking.

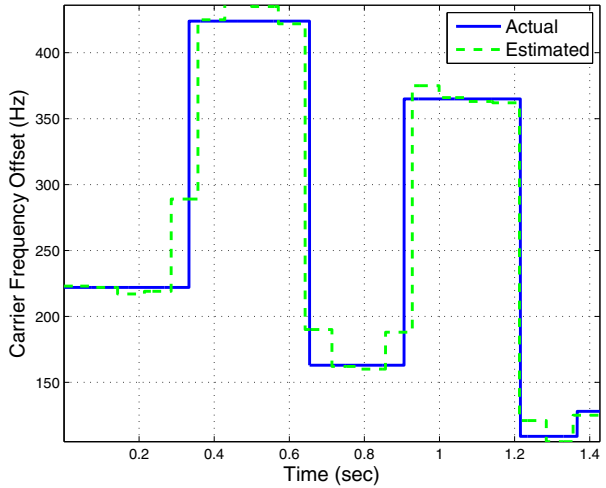

Fig. 7. Carrier frequency offset tracking.

\section{Simulation}

For tracking and adaptive channel estimation performance evaluation $20 \mathrm{MHz}$ bandwidth, 1200 data subcarriers and 2048 length FFT with $15 \mathrm{kHz}$ subcarrier spacing is assumed. Periodic pilot grid with spacing of 4 resource elements in both time and frequency direction and $21 \times 21$ LMMSE filter size is used. For tracking, we used a observation window of length $0.07 \mathrm{sec}$ i.e. after every $0.07 \mathrm{sec}$ the base station corrects the synchronization offsets per user and LMMSE filter adapts by sensing the statistics of the channel. Fig. 4 and Fig. 5 illustrate delay spread and BOF parameter tracking performance. Fig. 6 depicts Doppler spread tracking performance. Fig. 7 illustrates CFO tracking and Fig. 8 shows CFO tracking error PDF for different observation window lengths. In Fig. 8 expected error indicates bias which increases with window length and variance reflects the filter adaptation rate and noise effects. Large observation window gives more accurate estimates than the shorter window when there are small parameter fluctuations but in case of large parameter fluctuations its performance decreases. Fig. 9 shows the MSE channel estimation vs. SNR performance curves for perfect adaptive LMMSE filter (perfect parameter tracking), adaptive LMMSE filter with tracking errors, robust LMMSE filter (with maximum Doppler spread 


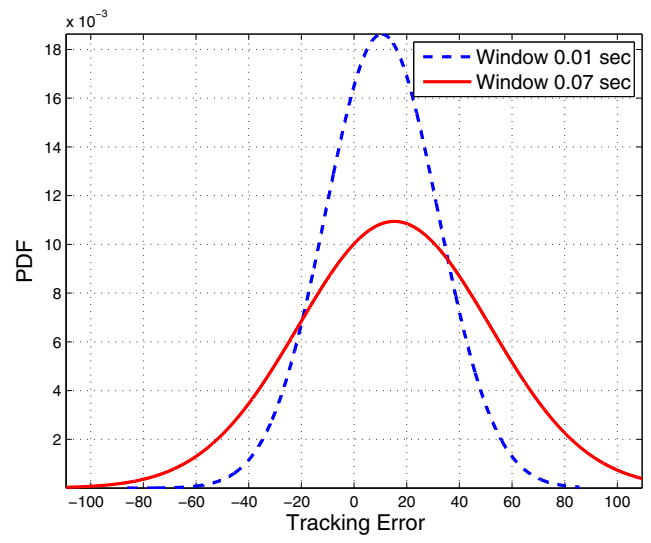

Fig. 8. CFO tracking error probability density function (PDF) for different window lengths.

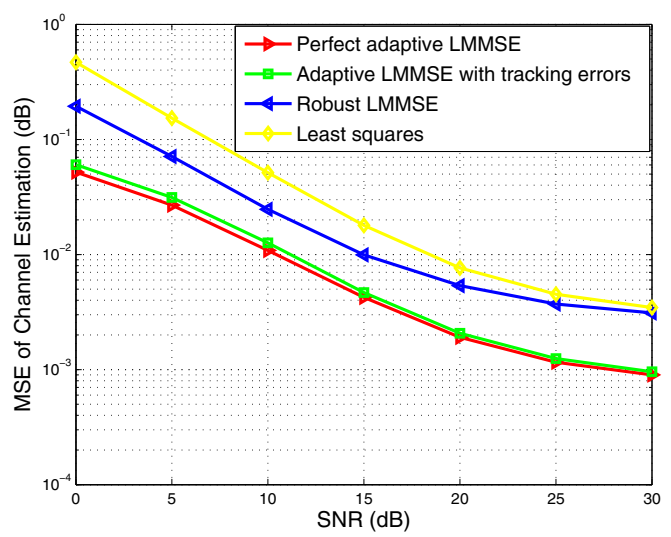

Fig. 9. Estimator performance with and without tracking errors.

set to $700 \mathrm{~Hz}$ and maximum delay spread set to cyclic prefix length) and least squares filtering. From the figure it is evident that our proposed 4-parametric adaptive LMMSE channel estimation scheme shows clear improvement over robust (static) LMMSE channel estimation and gain of more than $8 \mathrm{~dB}$ on average as compared to robust filtering is achieved with the stated simulation specifications.

\section{Discussion}

Modern mobile communication systems ensuring reliable transmission at high data rates along with varying channel statistics and synchronization offsets require high performance channel estimation. This requires adaptive channel estimation in order to exploit as much pilot correlation as possible. For MIMO-OFDMA transmission schemes 3D pilot correlation is available. The proposed $2 \mathrm{D}$ adaptive channel estimator can be extended to 3D in case of MIMOOFDMA transmission. Instead of fixed LMMSE filter size as used in the simulations, filter size adapted to tracked parameters can be used. To reduce the complexity of adaptive channel estimation rather than adaptive LMMSE filter coefficients calculation, an adaptive selection from one of the several precomputed filters can be made. To enable the adaptive choice of precomputed filter coefficients, the phase change due to synchronization offsets is compensated before the filtering (Fig. 3). In the paper we have tracked 4 parameters for improving channel estimation but in principal it is possible to track more number of parameters. Observation window length depends upon the coherence time of the channel therefore for fast varying channels a shorter observation window and for slowly varying channels a larger window is suggested.

\section{REFERENCES}

[1] B. J. C. L. Hanzo, M. Münster and T. Keller, OFDM and MC-CDMA. IEEE Press, 2003.

[2] Y. J. Ryu and D. S. Han, "Timing phase estimation overcoming rayleigh fading for OFDM systems," IEEE Trans. Consumer Electron., vol. 47, pp. 370-377, Aug. 2001.

[3] A. H. M. Makundi and T. Laakso, "An interpolation-based frequencysynchronization scheme for OFDM systems," Signal Processing Advances in Wireless Communications, pp. 151-155, 2005.

[4] P. Hoher, S. Kaiser, and P. Robertson, "Two-dimensional pilot-symbol aided channel estimation by wiener filtering," in Proc. IEEE Int. Conf. Acoustics, Speech, and Signal Processing, vol. 3, 1997, pp. 18451848.

[5] N. Wiener, Extrapolation, Interpolation, and Smoothing of Stationary Time Series. Wiley, 1949.

[6] V. Srivastava, C. K. Ho, P. H. W. Fung, and S. Sun, "Robust MMSE channel estimation in OFDM systems with practical timing synchronization," in Proc. IEEE Wireless Communications and Networking Conference (WCNC), vol. 2, 2004, pp. 711- 716.

[7] K. Jeong and J. Lee, "Low complexity channel tracking for adaptive MMSE channel estimation in OFDM," in IEEE CISS, 2007.

[8] C. Athaudage and A. Jayalath, "Enhanced MMSE channel estimation using timing error statistics for wireless OFDM systems," IEEE Transactions on Broadcasting, vol. 50, no. 4, pp. 369- 376, Dec. 2004.

[9] P. Bello, "Characterization of randomly time-variant linear channels," IEEE Trans. Communication Systems, vol. 11, no. 4, pp. 360-393, 1963.

[10] H. Minn, M. Zeng, and V. K. Bhargava, "On timing offset estimation for OFDM systems," IEEE Commun. Lett., pp. 242-244, July 2000.

[11] B. Yang, K. B. Letaief, R. S. Cheng, and Z. Cao, "Timing recovery for OFDM transmission," IEEE J. Select. Areas Commun., pp. 22782290, Nov 2000.

[12] C. Athaudage and A. Jayalath, "Delay-spread estimation using cyclic prefix in wireless OFDM systems," IEE Proc.-Commun, December 2004.

[13] K. Witrisal, Y. Kim, and R. Prasad, "A new method to measure parameters of frequency-selective radio channel using power measurements," IEEE Trans. Commun., vol. 49, no. 10, pp. 1788-1800, Oct 2001.

[14] T. Yücek and H. Arslan, "Time dispersion and delay spread estimation for adaptive OFDM systems," IEEE Trans. Vehicular Technology, vol. 57, no. 3, May 2008.

[15] T. Yücek, R. Tannious, and H. Arslan, "Doppler spread estimation for wireless OFDM systems," IEEE Advances in Wired and Wireless Comm., pp. 233-236, April 2005.

[16] Final Report on RF Channel Characterization, Joint Technical Committee (JTC) on Wireless Access, JTC(AIR)/93.09.23-238R2, September 1993.

[17] W. Song and J. Lim, "Pilot-symbol aided channel estimation for OFDM with fast fading channels," IEEE Transactions on Broadcasting, vol. 49, no. 4, pp. 398-402, December 2003. 